\title{
ColabIn: Modelo de Colaboração Interativa de Aula Fracionada para o Ensino Remoto na Educação Superior
}

\author{
Érica Mourão ${ }^{1}$, Marcel Dias $^{1}$, Edilson Pinheiro ${ }^{1}$, \\ José Viterbo $^{1}$, Cristiano Maciel $^{2}$ \\ ${ }^{1}$ Instituto de Computação - Universidade Federal Fluminense (UFF) \\ Niterói - RJ - Brasil \\ ${ }^{2}$ Instituto de Computação - Universidade Federal de Mato Grosso (UFMT) \\ Cuiabá - MT - Brasil \\ \{ericamourao, marceldias, edilsonpinheiro\}@id.uff.br \\ viterbodic.uff.br, cmacieleufmt.br
}

\begin{abstract}
Social distancing from the coronavirus pandemic accelerated changes in teaching and didactic-pedagogical practices. Presential teaching migrated to remote learning combined with the use of Information and Communication Technology to conduct classes. However, the lack of interaction between teachers and students can make learning tiresome and unmotivating. This work aims to propose a collaborative model of fractional classes with micro activities to improve the interaction between teachers and students. To evaluate the model, we carried out an experiment with Computer Science students. The results indicate greater ease of use, acceptance, and adoption intent than the traditional model in remote learning.
\end{abstract}

Resumo. O distanciamento social face a pandemia do coronavírus acelerou as transformações no ensino e nas práticas didático-pedagógicas. O ensino presencial migrou para o ensino remoto aliado ao uso de Tecnologia de Informação e Comunicação para realizar aulas. Entretanto, a falta da interação entre professor e alunos pode tornar a aprendizagem cansativa e pouco motivadora. $O$ objetivo deste trabalho é propor e avaliar um modelo colaborativo de aulas fracionadas com micro atividades para melhorar a interação entre professor e alunos. Para avaliar o modelo realizamos um experimento com alunos de Computação. Os resultados indicam grande facilidade de uso, aceitação e intenção de adoção em comparação com modelo tradicional no ensino remoto.

\section{Introdução}

A Organização Mundial de Saúde (OMS) declarou, em março de 2020, como status pandêmico a doença denominada COVID-19 [Bagatini et al. 2020], causada pelo vírus SARS-CoV-2 [Li et al. 2020], e defendeu o isolamento social como uma medida estratégica de combate à propagação do vírus [Usher et al. 2020]. Nas Instituições Públicas de Ensino Superior, diante da pandemia e das medidas de isolamento social, houve uma suspensão do ensino presencial. Entretanto, as instituições foram autorizadas a realizar o ensino remoto por meio das Tecnologias de Informação e Comunicação (TICs) [Santos et al. 2020]. 
Neste cenário de distanciamento social, esta nova realidade tem requerido adaptações nas metodologias de ensino-aprendizagem suportadas por tecnologias [Feitosa et al. 2020] e este é o momento oportuno para investigar novas práticas didático-pedagógicas [Pimentel and Carvalho 2020, Cardoso et al. 2021]. Diante do ensino remoto, a duração do tempo de aula foi uma das reflexões considerada pelos professores, seguida de preparar e apresentar conteúdo de aula utilizando recursos tecnológicos, e aprender novas formas de ensinar com a mediação tecnológica [Rodrigues 2020]. Por outro lado, os alunos universitários precisaram adaptar a rotina de estudos a partir do uso de tecnologias. Eles perceberam que o isolamento social teve impacto na sua rotina de estudos, no que tange aos aspectos da organização do tempo, preocupação e ansiedade [Bagatini et al. 2020].

Dentre os inúmeros desafios no ensino remoto, tais como: suporte tecnológico para acompanhamento das atividades remotas, mediação do ensino, e normas das ações e dos procedimentos para avaliações, um dos desafios identificados é a falta da percepção da interação entre professor e alunos, a qual é mais facilmente percebida no ensino presencial [Feitosa et al. 2020, Rodrigues 2020]. Essa falta de interação prejudica o rendimento e causa maior cansaço devido ao esforço para se manter por horas contínuas em frente a um computador, celular ou tablet para assistir às aulas [Feitosa et al. 2020]. Segundo Rodrigues (2020), uma aula expositiva presencial com duração de cinquenta minutos ou mais tende a ser cansativa, mas no ensino remoto essa duração é muito menos produtiva.

Portanto, considerando o exposto, o problema abordado neste trabalho são os desafios da interação entre professor e alunos e a duração de aula no ensino remoto. Com isso, identificou-se como questão de pesquisa: "O fracionamento de aulas visando a colaboração pode apoiar a interação e o engajamento entre professor e alunos na educação superior em Computação durante o ensino remoto?”.

Neste contexto, este trabalho tem como objetivo propor e avaliar um modelo de colaboração interativa de aulas fracionadas com micro atividades que pode apoiar a interação entre professor e alunos na educação superior em Computação durante o ensino remoto. Este modelo, que chamamos de ColabIn, foi proposto com base no Modelo 3C de Colaboração [Fuks et al. 2011] e beneficia-se de aulas e atividades intercaladas, com duração menor, para aumentar a interação e engajamento entre professor e alunos.

Para avaliar o modelo ColabIn, uma pesquisa explicativa de abordagem qualitativa e quantitativa foi conduzida através de um experimento [Wohlin et al. 2012] on-line com 29 participantes. Durante o experimento, apresentamos o cenário de aulas fracionadas com micro atividades e, após cada etapa os alunos de um curso de graduação em Computação responderam a questionários online. Utilizamos o método System Usability Scale (SUS) [Brooke 1996] para avaliar a usabilidade e a satisfação do modelo ColabIn e do modelo tradicional de ensino remoto e realizamos uma análise comparativa entre eles. Em seguida, utilizamos o Technology Acceptance Model 3 (TAM 3) [Venkatesh and Bala 2008] para avaliar a facilidade de utilidade, facilidade de uso, intenção de uso e satisfação do modelo ColabIn.

A colaboração e a interação em uma aula são fundamentais para um aprendizado efetivo. Nossos resultados demonstram que o modelo ColabIn, com aulas fracionadas e micro atividades, pode contribuir para que professores e alunos de Instituições de Ensino 
Superior tenham uma maior percepção da interação, dinamismo e engajamento, garantindo o ensino-aprendizagem mais efetivo neste momento de isolamento social.

A Seção 2 descreve os trabalhos relacionados, a Seção 3 apresenta a proposta de modelo, a Seção 4 apresenta o experimento, a Seção 5 apresenta os resultados e discussões. Por fim, a Seção 6 reporta as considerações finais do trabalho.

\section{Trabalhos Relacionados}

A busca por trabalhos relacionados foi realizada através de Revisão Sistemática da Literatura (RSL) que abordou modelos de colaboração para o ensino remoto. Foram utilizadas as diretrizes de Kitchenham e Charters (2007), que inclui o uso de Database Search para identificar estudos primários. Em seguida, utilizamos as diretrizes de Wohlin (2014), que usa Snowballing para busca por artigos relevantes de duas formas: busca nas referências dos artigos, chamada de Backward Snowballing (BS), e busca nas citações dos artigos, chamada de Forward Snowballing (FS). Essa estratégia de busca, que inclui Database Search seguida de Snowballing é considerada ampla e permite uma cobertura de artigos relevantes [Mourão et al. 2020]. O período de busca por estudos foi de 2015 até 2020 e dentre os resultados, identificou-se os que mais assemelham-se ao nosso problema e investigam propostas de modelos para maior satisfação e engajamento dos alunos no ensino remoto: Al-Rahmi et al. (2018), Alalwan et al. (2019), Alamri et al. (2020), Salam and Farooq (2020) e Abuhassna et al. (2020).

Os estudos de Al-Rahmi et al. (2018), Alalwan et al. (2019) e Alamri et al. (2020) investigam o uso de mídias sociais para aprendizagem colaborativa e engajamento de alunos de ensino superior. Eles utilizaram como método de avaliação do modelo fatores da Teoria do Construtivismo e do Technology Acceptance Model (TAM). Os resultados indicam que a aprendizagem colaborativa bem como a comunicação online nas redes sociais melhoram e enriquecem as atividades dos alunos.

A pesquisa de Salam and Farooq (2020) discutiu o impacto da qualidade da sociabilidade no uso de sistema de informação de aprendizagem colaborativa e a satisfação do usuário. Eles propõem um modelo teórico integrando fatores de sistema, satisfação e sociabilidade. Os resultados mostram que a qualidade da sociabilidade pode contribuir no domínio do aprendizado. O trabalho de Abuhassna et al. (2020) apresenta um modelo para melhorar as realizações acadêmicas e a satisfação dos alunos no uso de plataforma de aprendizagem online. Eles utilizaram a Teoria da Distância Transacional (TDT) para medir a satisfação e componentes da Teoria de Bloom para medir realizações acadêmicas dos alunos de cursos de ensino superior e sugerem investigações futuras utilizando o $T e$ chnology Acceptance Model (TAM). Os resultados indicam que o uso do modelo pode melhorar na tomada de decisão ao implementar plataformas de aprendizado online nas instituições.

O trabalho de Fuks et al. (2011) apresenta o Modelo 3C de Colaboração que possui três dimensões, chamadas de 3C: Comunicação, caracterizada pela troca de mensagens entre pessoas; Coordenação, caracterizada pelo gerenciamento de pessoas, atividades e recursos; e Cooperação, caracterizada pela atuação conjunta num espaço compartilhado para produção de artefatos ou informações [Pimentel et al. 2006]. Neste sentido, entendese que não há um trabalho sobre modelo de colaboração interativa para ensino remoto na pandemia COVID-19, com foco na interação em aulas fracionadas e micro atividades. 


\section{Modelo de Colaboração Interativa Proposta}

A proposta de modelo de colaboração interativa, que denominamos ColabIn, foi desenvolvida com base no Modelo 3C de Colaboração [Fuks et al. 2011]. Para a estruturação da proposta, definiu-se um cenário de aula no ensino remoto com o tema Pensamento Computacional na Educação. Em seguida, elaboramos o cenário da aula em um protótipo navegável, desenvolvido por meio da ferramenta on-line interativa de desenvolvimento colaborativo Padlet ${ }^{1}$ [Dewitt et al. 2015]. No protótipo, primeiro dividimos uma aula tradicional em aulas com duração menor, as quais chamamos de aulas fracionadas [Sousa et al. 2015, dos Santos Lima et al. 2019]. Depois, definimos micro atividades, que são tarefas curtas, relacionadas respectivamente a cada aula fracionada. Na Figura 1 do protótipo, podemos observar a primeira fração de aula, seguida de sua micro atividade 1. Em seguida, a segunda fração de aula, seguida de sua micro atividade 2. Por último, a terceira fração de aula, seguida de sua micro atividade 3. As aulas fracionadas foram definidas com duração de 7 minutos e as micro atividades com duração de 3 minutos.

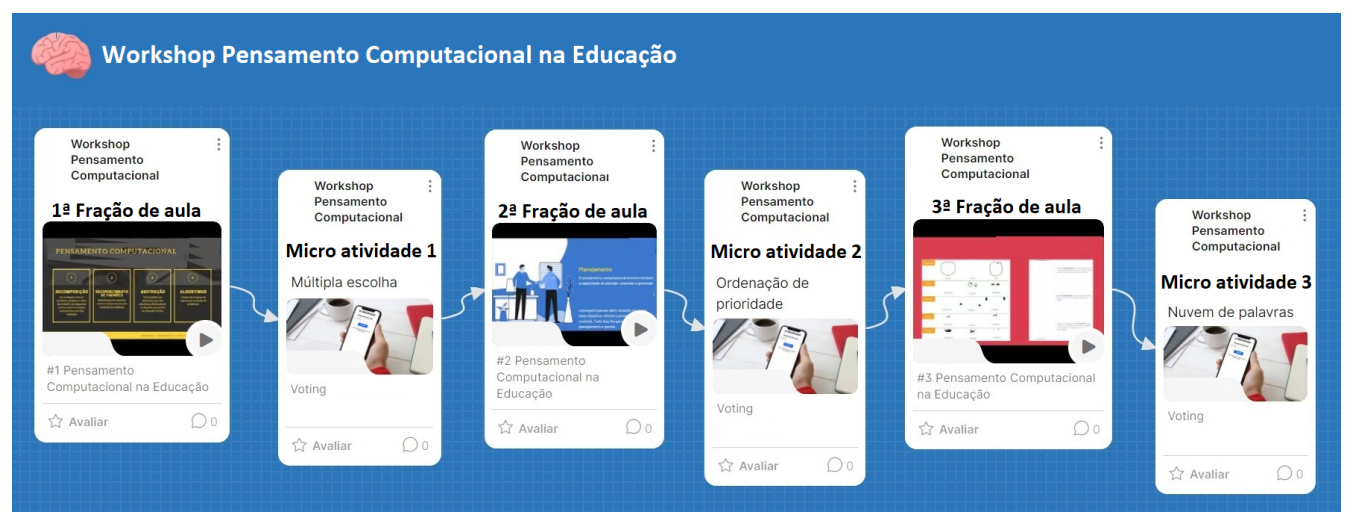

Figura 1. Protótipo de aulas fracionadas e micro atividades

Um aspecto relevante do modelo ColabIn é promover o aprendizado do conteúdo das aulas a cada término de uma fração de aula, permitindo assim um aprendizado incremental, contínuo e uma melhor percepção da interação colaborativa entre professor e alunos. Um outro aspecto do modelo ColabIn são as micro atividades aplicadas por meio de atividades colaborativas. Isso permite maior dinamismo e interação entre professor e alunos, reduzindo o declínio cognitivo. No modelo ColabIn, a coordenação, a cooperação e a comunicação durante as aulas fracionadas e micro atividades, são determinantes para promover a motivação e a interação durante o ensino remoto.

O processo do modelo ColabIn foi desenhado utilizando a notação de modelagem de processo de negócios (BPMN)[White 2004] e possui dois atores: professor e aluno, conforme observado na Figura 2. Para dar início ao processo o professor cria o cenário de aula em "Criar cenário da aula", onde o professor identifica o tema da aula, organiza o material de aula e configura o Ambiente Virtual de Aprendizagem (AVA)[Maciel 2013]. Após o cenário criado e pronto para iniciar a aula, o professor apresenta a primeira fração de aula aos alunos em "Apresentar fração de aula". Os alunos assistem à fração de aula em "Assistir fração de aula" que tem a duração de 07 minutos. Ao seu término, o professor apresenta aos alunos a primeira micro atividade em "Apresentar micro atividade"

\footnotetext{
${ }^{1}$ https://www.padlet.com/
} 
relacionada a primeira fração de aula. Os alunos têm até 3 minutos para realizar a micro atividade em "Realizar micro atividade". Após seu término, o professor apresenta e discute a solução do problema com seus alunos em "Apresentar solução da micro atividade". As aulas são no formato síncrono, mas podem ser no assíncrono, pois as aulas são disponibilizadas no AVA.

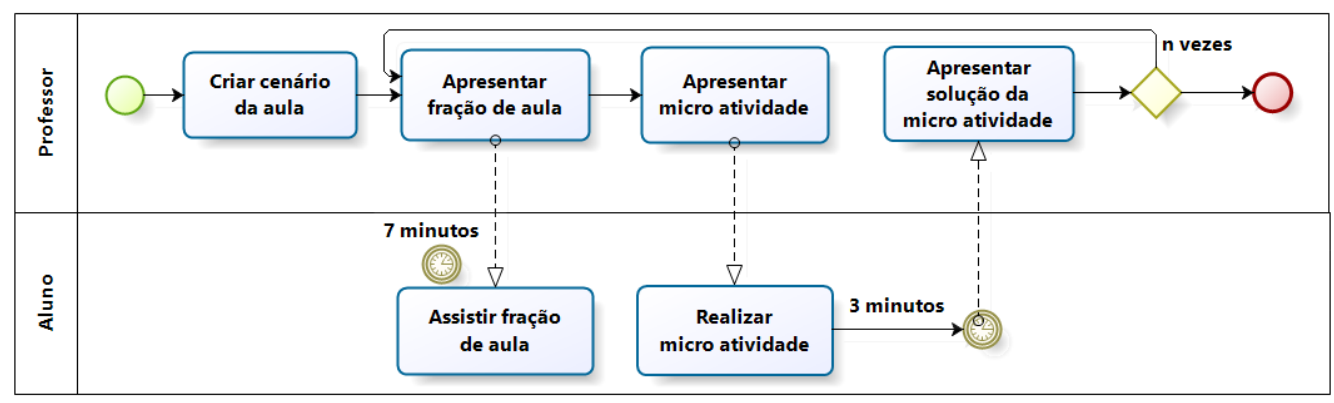

Figura 2. Processo do modelo de colaboração interativa Colabln

Após a apresentação da solução da primeira micro atividade, referente a primeira fração de aula, as etapas do processo podem ser repetida "n vezes" de acordo com o tempo total pré-definido para a aula, a partir da apresentação de uma nova fração de aula em "Apresentar fração de aula". Por exemplo, aplicando uma aula de 30 minutos no modelo proposto, teríamos 3 aulas fracionadas de 7 minutos, sendo cada uma seguida de uma micro atividade de 3 minutos. Para avaliar o modelo proposto, um experimento foi realizado e é apresentado na próxima seção.

\section{Experimento}

Com a finalidade de verificar a viabilidade da proposta de modelo ColabIn, um experimento foi realizado utilizando um cenário de aula remota. O estudo experimental foi realizado durante a pandemia de COVID-19 em novembro de 2020 e todas as etapas foram realizadas on-line no Google Meet. Alunos voluntários de graduação em Computação de uma Instituição de Ensino Superior foram convocados para participar do experimento durante o "Workshop de Pensamento Computacional na Educação". O objetivo foi identificar a satisfação, usabilidade, utilidade, facilidade de uso, aceitação e intenção de uso do modelo ColabIn, através de questionários on-line durante uma aula remota.

O método System Usability Scale (SUS) [Brooke 1996] foi utilizado para avaliar a satisfação e a usabilidade durante a interação com o modelo ColabIn. Além disso, utilizou-se o SUS para avaliar o modelo tradicional de aula remota e com isso realizar uma análise comparativa com o modelo ColabIn. O método Technology Acceptance Model 3 (TAM 3) [Venkatesh and Bala 2008] foi utilizado para avaliar a utilidade, uso, aceitação, intenção de uso e satisfação do modelo ColabIn.

Nos questionários on-line foram utilizadas questões com respostas objetivas que consideraram a escala Likert [Brooke 1996], que possibilita avaliar as perguntas entre extremos, por exemplo de "concorda fortemente" até "discorda fortemente", considerando 5 níveis de concordância. O método SUS utilizou somente questões objetivas baseadas na Escala Likert. Entretanto, o TAM utilizou a Escala Likert e questões discursivas.

Durante a execução do experimento foram utilizados como artefatos 06 (seis) questionários on-line desenvolvidos na ferramenta Google Forms, sendo: (i) questionário 
para o termo de consentimento livre e esclarecido; (ii) questionário para a caracterização dos participantes; (iii) questionário para aplicação do método SUS para avaliar o modelo ColabIn (iv) questionário para aplicação do método SUS para avaliar o modelo tradicional de aula remota; e (iv) questionário para aplicação do TAM 3 para avaliar o ColabIn.

Foi criado um cenário de ensino remoto e uma aula com o tema Pensamento Computacional na Educação foi ministrada, com base no modelo ColabIn. O objetivo da aula foi o aluno aprender a desenvolver sua capacidade criativa lógica e estratégica para ser capaz de transformar um problema complexo em algo fácil de ser compreendido e resolvido com base computacional. O conteúdo da aula foi dividido em três aulas fracionadas, com duração de sete minutos cada uma, e suas micro atividades com duração de 3 minutos.

O experimento foi definido em três etapas: (1) Preparação, (2) Execução, e (3) Validação dos dados. 1) Preparação: primeiro, um e-mail convite foi enviado aos alunos de ensino superior na área de Computação para participar voluntariamente do experimento durante o Workshop de Pensamento Computacional na Educação. Após a inscrição no workshop, eles foram redirecionados para um questionário contendo um Termo de Consentimento Livre e Esclarecido e outro contendo o Questionário de Perfil do Participante; 2) Execução: as frações de aula foram apresentadas pelo professor aos alunos de ensino superior e as micro atividades foram intercaladas entre as frações de aula. Após o cenário de aula, os alunos responderam aos questionários on-line SUS e TAM; 3) Validação de Dados: após o experimento, os dados foram anonimizados e validados para garantir a confiabilidade. Na próxima seção os resultados obtidos são apresentados e discutidos.

\section{Resultados}

De um total de 33 alunos de ensino superior em Computação inscritos, 29 participaram do experimento, sendo $24,1 \%$ entre 18 e 20 anos, $69 \%$ com a faixa etária entre 21 e 30 anos e 6,9\% acima de 30 anos. Dentre os participantes, todos são alunos de graduação em Computação de uma Universidade Pública e 96,6\% informaram que têm acesso a internet na residência. Quanto à participação em aula remota, 100\% dos participantes indicaram que já participaram de uma aula remota, sendo que $75,9 \%$ usou o dispositivo notebook, $13,8 \%$ usou desktop e 10,3\% usou o smartphone. Diante desse resultado, podese considerar que o nível de conhecimento e habilidades dos sujeitos contribuirão para a avaliação do modelo proposto ColabIn.

Quando questionados sobre há quanto tempo eles participam de aula remota, $79,3 \%$ informaram que participam de aula remota há menos de 6 meses, $13,8 \%$ entre 6 meses e 1 ano, a mais de 2 ano 6,9\%. Quanto à frequência que eles participam de aulas remotas, 41,4\% informaram que participam 3 vezes por semana, 27,6\% participam 2 vezes por semana, 24,1\% participam 1 vez por semana, 6,9\% participam 4 vezes ou mais por semana. Quanto ao tipo de plataforma de colaboração de aula remota, os participantes podiam marcar mais uma opção de resposta, onde 82,8\% utilizam a plataforma de colaboração de aula remota SIGAA - Sistema Integrado de Gestão de Atividades Acadêmicas, 41,4\% afirmaram Google Classroom, 6,9\% Moodle. Quanto à resposta do que o participante acha importante para se manter motivado na aula remota, 37,93\% informaram razoavelmente relevante, $27,59 \%$ muito relevante, e 34,48\% extremamente relevante as atividades baseadas em problema.

A fim de verificar a usabilidade e satisfação do modelo ColabIn com aulas fraci- 
onadas e micro atividades, foram abordadas questões do SUS. A Tabela 1 apresenta os resultados da avaliação dos participantes em relação a usabilidade e satisfação do modelo ColabIn. Ela mostra resultados de discordância até concordância em cinco níveis: Discorda Fortemente (DF), Discorda (D), Neutro (N), Concorda (C), Concorda Fortemente (CF). Podemos observar que $82,76 \%$ dos participantes $(41,38 \% \mathrm{C}$ e $41,38 \% \mathrm{CF})$ gostariam de ter aulas neste novo modelo frequentemente. Quanto a complexidade na participação, 75,86\% dos participantes (31,03\% DF e 44,83\% D) indicaram ser capazes de utilizar o modelo ColabIn. Quando questionados sobre a facilidade em participar do modelo ColabIn, $86,21 \%$ dos participantes $(48,28 \% \mathrm{C}$ e $37,93 \% \mathrm{CF})$ indicaram que o modelo foi satisfatório. Com isso, pode-se notar que a percepção de satisfação é positiva.

Tabela 1. Avaliação SUS do Modelo Colabln

\begin{tabular}{|c|c|c|c|c|c|}
\hline Questão Avaliada & DF (\%) & $\mathbf{D}(\%)$ & $\mathbf{N}(\%)$ & $\mathrm{C}(\%)$ & CF (\%) \\
\hline Gostaria de ter aulas neste novo modelo de aula online frequentemente & 0,00 & 10,34 & 6,90 & 41,38 & 41,38 \\
\hline Achei complexo participar deste novo modelo de aula online & 31,03 & 44,83 & 6,90 & 13,79 & 3,45 \\
\hline Achei fácil participar deste novo modelo de aula online & 3,45 & 0,00 & 10,34 & 48,28 & 37,93 \\
\hline Precisaria de mais explicações para um melhor entendimento deste novo modelo de aula online & 24,14 & 31,03 & 27,59 & 13,79 & 3,45 \\
\hline Achei que o conteudo e atividades deste novo modelo de aula online estavam bem relacionados & 3,45 & 6,90 & 0,00 & 55,17 & 34,48 \\
\hline Achei que havia muita inconsistência no novo modelo de aula online. & 48,28 & 31,03 & 10,34 & 10,34 & 0,00 \\
\hline Imagino que a maioria das pessoas poderiam aprender melhor nesse novo modelo de aula online & 0,00 & 3,45 & 17,24 & 41,38 & 37,93 \\
\hline Achei este novo modelo de aula online incompleto & 37,93 & 31,03 & 17,24 & 10,34 & 3,45 \\
\hline Me senti muito seguro participando deste novo modelo de aula online & 6,90 & 6,90 & 17,24 & 48,28 & 20,69 \\
\hline Precisaria experimentar mais aulas neste novo modelo de aula online para avaliar melhor & 10,34 & 17,24 & 24,14 & 37,93 & 10,34 \\
\hline
\end{tabular}

Quanto a necessidade de mais explicações para melhor entender o modelo ColabIn, $55,17 \%(24,14 \%$ DF e 31,03\% D) indicaram que o esclarecimento foi satisfatório, entretanto, outros $27,59 \%$ informaram que a explicação atendeu parcialmente. É possível verificar que quanto ao conteúdo e atividades estarem relacionados, 89,65\% (55,17\% C e 34,48\% CF) afirmaram positivamente. Quando questionados sobre a existência de inconsistência no modelo, 79,31\% (48,28\% DF e 31,03\% D) informaram a percepção de coerência. Quanto ao nível de aprendizado da maioria das pessoas, 79,31\% (41,38\% C e $37,93 \%$ CF) afirmaram ser um melhor modelo ColabIn para aprender. Diante do exposto, pode-se notar a percepção do engajamento no modelo ColabIn.

Quanto ao modelo ser incompleto, é possível constatar que apenas 13,79\% dos participantes $(10,34 \%$ C e 3,45\% CF) indicaram que o modelo ColabIn não está completo. Em relação a segurança na participação do uso do modelo, 68,97\% (48,28\% C e 20,69\% $\mathrm{CF}$ ) responderam se sentir seguros em participar do modelo ColabIn. Entretanto, outros $17,24 \%$ avaliaram de maneira imparcial. Quanto a necessidade de experimentar mais aulas no modelo ColabIn, para poder avaliar melhor ele, $48,27 \%$ (37,93\% C e 10,34\% $\mathrm{CF}$ ) afirmam essa necessidade. Com isso, pode-se notar a completude do modelo ColabIn e a intenção de uso.

A fim de verificar a usabilidade e satisfação do modelo tradicional de aula remota, durante o experimento foi solicitado aos participantes responder o questionário SUS de acordo com o seu conhecimento prévio e experiência de participação em aulas remotas tradicionais. A Tabela 2 apresenta os resultados da avaliação dos participantes em relação a usabilidade e satisfação do modelo tradicional. Podemos observar que 44,83\% dos participantes $(37,93 \%$ C e $6,90 \% \mathrm{CF})$ gostariam de ter aulas remotas no modelo tradicional frequentemente. Quanto a complexidade na participação, 68,97\% dos participantes $(24,14 \%$ DF e $44,83 \%$ D) indicaram ser capazes de utilizar o modelo tradicional. Quando questionados sobre a facilidade em participar do modelo de aula, 68,97\% (44,83\% C e 
24,14\% CF) dos participantes indicaram que o modo tradicional foi satisfatório. Com isso, observa-se a familiaridade com o modelo tradicional de aula remota.

Tabela 2. Avaliação SUS do Modelo Tradicional de Ensino Remoto

\begin{tabular}{|c|c|c|c|c|c|}
\hline Questão Avaliada & DF (\%) & $\mathbf{D}(\%)$ & $\mathbf{N}(\%)$ & $\mathrm{C}(\%)$ & CF (\%) \\
\hline Gostaria de ter aulas neste modelo tradicional de aula online frequentemente. & 6,90 & 20,69 & 27,59 & 37,93 & 6,90 \\
\hline Achei complexo participar deste modelo tradicional de aula online. & 24,14 & 44,83 & 13,79 & 17,24 & 0,00 \\
\hline Achei fácil participar deste modelo tradicional de aula online. & 10,34 & 6,90 & 13,79 & 44,83 & 24,14 \\
\hline Precisaria de mais explicações para um melhor entendimento deste modelo tradicional de aula online. & 20,69 & 31,03 & 20,69 & 17,24 & 10,34 \\
\hline Achei que o conteúdo e atividades deste modelo tradicional de aula online estavam bem relacionados. & 3,45 & 31,03 & 20,69 & 37,93 & 6,90 \\
\hline Achei que havia muita inconsistência no modelo tradicional de aula online. & 13,79 & 37,93 & 24,14 & 20,69 & 3,45 \\
\hline Imagino que a maioria das pessoas poderiam aprender melhor neste modelo tradicional de aula online. & 27,59 & 27,59 & 24,14 & 13,79 & 6,90 \\
\hline Achei este modelo tradicional de aula online incompleto. & 17,24 & 20,69 & 20,69 & 27,59 & 13,79 \\
\hline Me senti muito seguro participando deste modelo tradicional de aula online. & 10,34 & 20,69 & 27,59 & 31,03 & 10,34 \\
\hline Precisaria experimentar mais aulas neste modelo tradicional de aula online para avaliar melhor. & 17,24 & 34,48 & 24,14 & 24,14 & 0,00 \\
\hline
\end{tabular}

Quanto a necessidade de mais explicações para melhor entender o modelo tradicional de aula remota, $51,72 \%(20,69 \%$ DF e 31,03\% D) indicaram que o esclarecimento foi satisfatório, entretanto, outros 20,69\% informaram que a explicação atendeu parcialmente. É possível verificar que quanto ao conteúdo e atividades estarem relacionados, $44,83 \%$ (37,93\% C e 6,9\% CF) afirmaram positivamente. Quando questionados sobre a existência de inconsistência no modelo tradicional de aula remota, 51,72\% (13,79\% DF e 37,93\% D) informaram a percepção de coerência. Quanto ao nível de aprendizado da maioria das pessoas, $55,18 \%(27,59 \%$ DF e $27,59 \%$ D) informaram não ser o melhor modelo.

Quanto ao modelo tradicional de aula remota ser incompleto, é possível constatar que 41,38\% (27,59\% C e 13,79\% CF) dos participantes indicaram que o modelo não está completo. Em relação a segurança na participação do uso do modelo, 41,37\% (31,03\% $\mathrm{C}$ e $10,34 \% \mathrm{CF}$ ) se sentiram seguros em participar do modelo tradicional. Quanto a necessidade de experimentar mais aulas no modelo tradicional de aula remota, para poder avaliar melhor ele, $51,72 \%(17,24 \%$ DF e $34,48 \%$ D) não necessitam experimentar mais aulas para uma melhor avaliação.

O resultado da análise comparativa do SUS para avaliar usabilidade indica uma maior satisfação para o modelo ColabIn, que obteve um score de 54.91 [Brooke 1996], em comparação com o resultado do SUS aplicado ao modelo de aula tradicional, que apresentou um score de 47.77 pontos. Contudo, realizamos teste de Wilcoxon [Cuzick 1985] pareado para amostras do SUS por participantes, que é usado para identificar qual das amostras apresentou significância estatística, e utilizamos um intervalo de confiança de 95\%. Obtivemos o p-value e concluímos que não há diferença.

A fim de verificar a percepção de utilidade, uso, aceitação e intenção de uso do modelo ColabIn foram abordadas questões do TAM. Os resultados da Avaliação da Percepção da Utilidade mostram, conforme Figura 3, que 93,10\% dos participantes $(34,48 \%$ CF e 58,62\% C) informaram que o modelo ColabIn permitiria realizar tarefas mais rapidamente. Em relação a melhoria de desempenho nos estudos, 72,41\% (13,79\% $\mathrm{CF}$ e $58,62 \% \mathrm{C}$ ) afirmaram positivo. Em relação a aumento da produtividade, $86,21 \%$ $(17,24 \%$ CF e $68,97 \%$ C) apresentaram percepção positiva seguida de $72,41 \%$ (20,69\% CF e $51,72 \%$ C) melhoria na produtividade, aumentando a eficácia nos estudos. Quanto a melhorar a eficácia e ser mais útil, respectivamente, $82,76 \%(24,14 \%$ CF e $58,62 \%$ C) e $96,55 \%$ (31,03\% CF e 65,52\% C) informaram alta satisfação e utilidade. Com isso, nota-se alta percepção de utilidade do modelo ColabIn. 


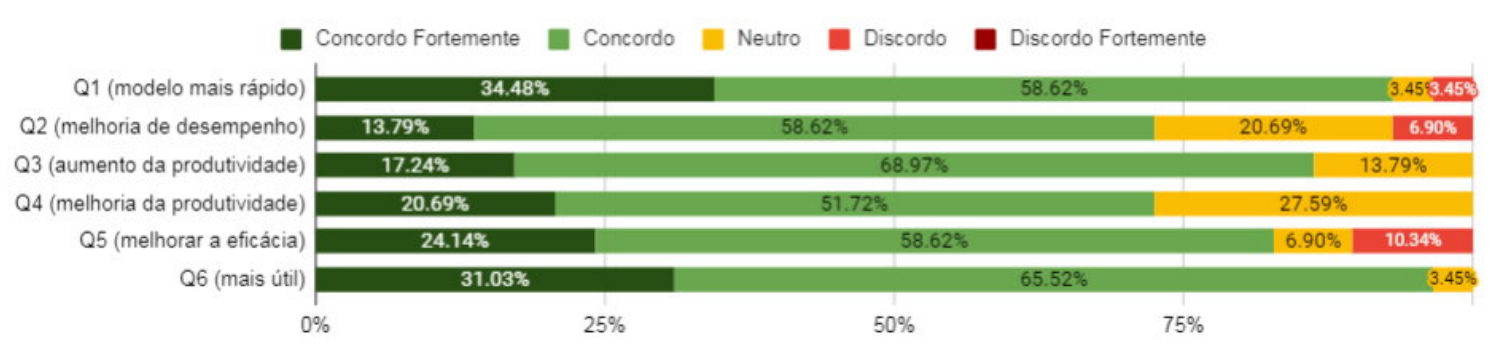

Figura 3. Percepção de Utilidade do Modelo Colabln

Os resultados da Avaliação da Percepção do Uso mostram, conforme Figura 4 que $96,55 \%$ dos participantes $(31,03 \% \mathrm{CF}$ e $65,52 \mathrm{C} \%)$ informaram que o modelo ColabIn é fácil de aprender. Quanto a facilidade de realizar os estudos, 72,41\% (20,69\% $\mathrm{CF}$ e $51,72 \% \mathrm{C}$ ) afirmaram ser fácil de realizar, entretanto $27,59 \%$ se mantiveram imparciais. Em relação a ser flexível de interagir com o modelo ColabIn, 86,21\% (27,59\% $\mathrm{CF}$ e 58,62\% C) avaliaram positivamente. Quanto a ser fácil de lembrar e fácil de usar, respectivamente, $89,65 \%(37,93 \% \mathrm{CF}$ e $51,72 \% \mathrm{C})$ e $93,10 \%(41,38 \% \mathrm{CF}$ e $51,72 \% \mathrm{C})$ informaram alta facilidade de usar. Com isso, ressalta-se alta percepção de uso do modelo ColabIn.

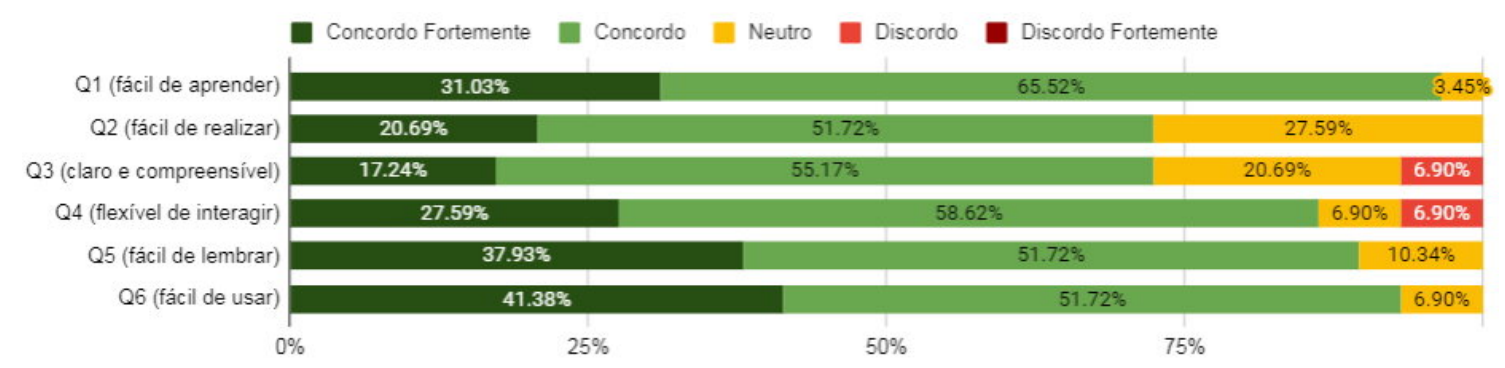

Figura 4. Percepção de Uso do Modelo Colabln

Os resultados da Avaliação da Intenção de Uso mostram, conforme Figura 5, que 93,10\% (34,48\% CF e 58,62\% C) pretendem usar o modelo ColabIn. Quanto a previsão de uso, $100 \%$ dos participantes avaliaram positivamente. Por último, $68,97 \%(41,38 \% \mathrm{CF}$ e $27,59 \%$ C) informaram que pretendem usar nos próximos 6 meses, entretanto 27,59\% mostram imparcialidade. Com isso, ressalta-se alta intenção de uso do modelo ColabIn.

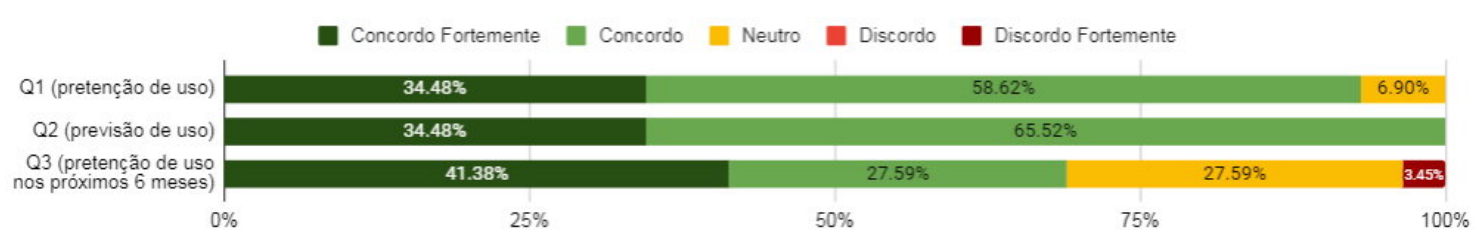

Figura 5. Intenção de Uso do Modelo Colabln

Os resultados da Avaliação da Satisfação de Uso mostram, conforme Figura 6, que $89,65 \%$ (37,93\% CF e 51,72 C) dos participantes afirmaram ser agradável de usar, e $89,66 \%$ (41,38\% CF e 48,28\% C) informaram ter o processo de uso do modelo ColabIn agradável. Quanto a ser divertido de usar, 62,07\% (34,48 CF e 27,59\% C) afirmaram ser divertido mas $34,48 \%$ mostraram imparcialidade. Com isso, nota-se a alta satisfação de uso do modelo ColabIn. 


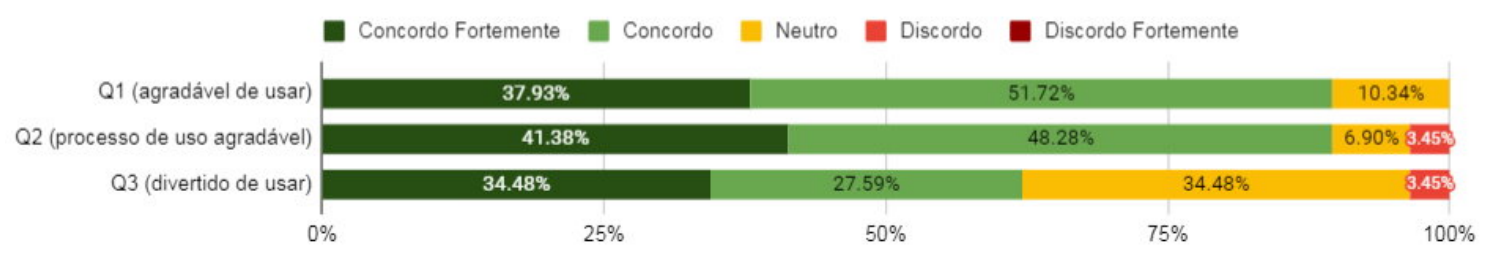

Figura 6. Satisfação de Uso do Modelo Colabln

A fim de verificar a percepção do engajamento do Modelo ColabIn, utilizamos os dados qualitativos coletados através do Questionário TAM. Classificamos as respostas qualitativas em positivas, neutras ou negativas, sobre como os Participantes $(\mathrm{P})$ consideram o modelo ColabIn e o modelo tradicional de aulas remotas.

Algumas citações fornecidas pelos participantes consideram o modelo ColabIn de forma positiva, como por exemplo, interessante, interativo, eficiente, agradável: "Motivador, prendeu minha atenção. Me fez prestar mais atenção no conteúdo ministrado, pois logo em seguida eu já teria que responder algo relacionado ao que o professor falou. Achei interessante." - P9; "Muito bom, gostei bastante da interações que fizemos. Você vê o conteúdo e já o pratica, isso é muito bom pra fixação." - P13; "Fácil de compreender, não torna cansativo o fato de serem vídeos curtos , melhor absorção do conteúdo.” $P 25$. Por outro lado, foi possível verificar algumas citações que consideram o modelo de aulas tradicionais de forma negativa, prevalecendo o termo cansativo: "Cansativo, Longo, Não dinâmico e emburrecedora." - P7; "Eficientes, nada de muito bons e nem ruins" $P 1$. De acordo com a análise qualitativa dos resultados da percepção do engajamento e compreensão, identifica-se uma alta aceitação do modelo ColabIn.

\section{Considerações Finais}

Neste trabalho foi possível comprovar a eficácia do fracionamento de aulas com micro atividades visando a colaboração e interação entre professor e alunos. Propôs-se o modelo ColabIn e avaliou-se usabilidade, satisfação e intenção de uso do ColabIn através de um experimento com alunos de graduação em Computação em Instituição de Ensino Superior.

Os resultados apresentados neste trabalho indicam que a satisfação no uso (SUS) do modelo ColabIn teve uma avaliação fortemente positiva e maior do que o modelo tradicional de ensino remoto. Em relação às percepções de utilidade, facilidade de uso, intenção de uso e satisfação (TAM), os resultados demonstram que o modelo ColabIn é mais vantajoso apresentando alta viabilidade de uso.

Nesse contexto, os resultados indicam que o modelo ColabIn pode melhorar a experiência de ensino aprendizagem, aumentar a satisfação e o engajamento, impactando positivamente o desempenho dos alunos. Este estudo comprovou que os resultados mostram-se promissores, apesar da ameaça à validade [Wainer et al. 2007] da amostra e limitações como: validar em uma turma, em Computação e o uso de um único AVA.

Observa-se que o modelo ColabIn foi avaliado como prático, simples, dinâmico e interativo, contudo, como trabalhos futuros, pretendemos realizar novos experimentos para avaliar o modelo levando em consideração alunos do ensino básico, graduação e pósgraduação. Espera-se, portanto, que este trabalho possa contribuir como um modelo de ensino remoto inovador que proporciona maior interação e aprendizado aos alunos. 


\section{Referências}

Abuhassna, H., Al-Rahmi, W. M., Yahya, N., Zakaria, M. A. Z. M., Kosnin, A. B. M., and Darwish, M. (2020). Development of a new model on utilizing online learning platforms to improve students' academic achievements and satisfaction. International Journal of Educational Technology in Higher Education, 17(1):1-23.

Al-Rahmi, W. M., Alias, N., Othman, M. S., Marin, V. I., and Tur, G. (2018). A model of factors affecting learning performance through the use of social media in malaysian higher education. Computers \& Education, 121:59-72.

Alalwan, N., Al-Rahmi, W. M., Alfarraj, O., Alzahrani, A., Yahaya, N., and Al-Rahmi, A. M. (2019). Integrated three theories to develop a model of factors affecting students' academic performance in higher education. IEEE Access, 7:98725-98742.

Alamri, M. M., Almaiah, M. A., and Al-Rahmi, W. M. (2020). The role of compatibility and task-technology fit (ttf): On social networking applications (snas) usage as sustainability in higher education. IEEE Access, 8:161668-161681.

Bagatini, D., Soares, R., Barros, E., and Silveira, G. (2020). Estudo virtual em tempos de distanciamento: uma análise sobre o impacto do isolamento social na rotina de estudos de universitários. In Anais do XXXI Simpósio Brasileiro de Informática na Educação, pages 792-801, Porto Alegre, RS, Brasil. SBC.

Brooke, J. (1996). SUS - a quick and dirty usability scale. Usability evaluation in industry, 189(194):4-7.

Cardoso, M. J. C., Almeida, G. D. S., and Silveira, T. C. (2021). Formação continuada de professores para uso de tecnologias da informação e comunicação (tic) no brasil. Revista Brasileira de Informática na Educação, 29:97-116.

Cuzick, J. (1985). A wilcoxon-type test for trend. Statistics in medicine, 4(1):87-90.

Dewitt, D., Alias, N., and Siraj, S. (2015). Collaborative learning: Interactive debates using padlet in a higher education institution. International Educational Technology Conference.

dos Santos Lima, J., de Figueirêdo, A. M. T. A., da Silva Araújo, V. M., Sales, F. R. P., and Tavares, M. J. F. (2019). Utilização de ferramentas didáticas no ensino de química visando aprimorar o processo de ensino-aprendizagem. International Journal Education and Teaching (PDVL) ISSN 2595-2498, 2(1):208-221.

Feitosa, M. C., de Souza Moura, P., Ramos, M. d. S. F., and Lavor, O. P. (2020). Ensino remoto: $\mathrm{O}$ que pensam os alunos e professores? In Anais do V Congresso sobre Tecnologias na Educação, pages 60-68. SBC.

Fuks, H., Raposo, A. B., Gerosa, M. A., Pimentel, M., Filippo, D., and Lucena, C. d. (2011). Teorias e modelos de colaboração. Sistemas colaborativos, pages 16-33.

Kitchenham, B. and Charters, S. (2007). Guidelines for performing systematic literature reviews in software engineering. Technical Report EBSE 2007-001. Keele University and Durham University Joint Report.

Li, R., Pei, S., Chen, B., Song, Y., Zhang, T., Yang, W., and Shaman, J. (2020). Substantial undocumented infection facilitates the rapid dissemination of novel coronavirus (sarscov-2). Science, 368(6490):489-493. 
Maciel, C. (2013). Educação a distância: ambientes virtuais de aprendizagem, volume 1, page 259. Universidade Federal de Mato Grosso, $1^{\text {a }}$ edition.

Mourão, E., Pimentel, J. F., Murta, L., Kalinowski, M., Mendes, E., and Wohlin, C. (2020). On the performance of hybrid search strategies for systematic literature reviews in software engineering. Information and Software Technology, 123:106294.

Pimentel, M. and Carvalho, F. d. S. P. (2020). Princípios da educação online: para sua aula não ficar massiva nem maçante. SBC Horizontes. Disponível em: http://horizontes.sbc.org.br/index.php/2020/05/principios-educacaoonlinel.[Acesso 05 Jul. 2020].

Pimentel, M., Gerosa, M. A., Filippo, D., Raposo, A., Fuks, H., and Lucena, C. J. P. d. (2006). Modelo 3c de colaboração para o desenvolvimento de sistemas colaborativos. Anais do III Simpósio Brasileiro de Sistemas Colaborativos, pages 58-67.

Rodrigues, A. (2020). Ensino remoto na educação superior: desafios e conquistas em tempos de pandemia. SBC Horizontes. Disponível em: http://horizontes.sbc.org.br/index. php/2020/06/ensino-remoto-na-educacao-superior/.[Acesso $05 \mathrm{Jul}$. 2020].

Salam, M. and Farooq, M. S. (2020). Does sociability quality of web-based collaborative learning information system influence students' satisfaction and system usage? International Journal of Educational Technology in Higher Education, 17:1-39.

Santos, C., Paillard, G., Moreira, L., Filho, F. R. S., and Coutinho, E. (2020). Uma análise qualitativa sobre atividades remotas em disciplinas no período de isolamento social. In Anais do XXXI Simpósio Brasileiro de Informática na Educação, pages 292-301, Porto Alegre, RS, Brasil. SBC.

Sousa, A., Silva, S., Raiol, A. A., Sarges, J., and Bezerra, F. d. L. (2015). O universo lúdico da programação de computadores com logo no ensino fundamental. In XXIII Workshop sobre Educação em Informática.

Usher, K., Bhullar, N., and Jackson, D. (2020). Life in the pandemic: Social isolation and mental health. Journal of clinical nursing, 29(15-16):2756-2757.

Venkatesh, V. and Bala, H. (2008). Technology acceptance model 3 and a research agenda on interventions. Decision sciences, 39(2):273-315.

Wainer, J. et al. (2007). Métodos de pesquisa quantitativa e qualitativa para a ciência da computação. Atualização em informática, 1(221-262):32-33.

White, S. A. (2004). Introduction to bpmn. Ibm Cooperation, 2(0):0.

Wohlin, C. (2014). Guidelines for snowballing in systematic literature studies and a replication in software engineering. In Proceedings of the 18th International Conference on Evaluation and Assessment in Software Engineering, pages 1-10.

Wohlin, C., Runeson, P., Höst, M., Ohlsson, M. C., Regnell, B., and Wesslén, A. (2012). Experimentation in Software Engineering. Springer Science \& Business Media. 